\title{
SATISFAÇÃO DO ATENDIMENTO FISIOTERAPÊUTICO DISPONIBILIZADO PELO NÚCLEO DE APOIO À SAÚDE DA FAMÍLIA
}

Keren Cristina Rodrigues Soares, Juliana Gonçalves Silva de Mattos.

\section{RESUMO}

Pergunta: Avaliar a satisfação dos pacientes quanto aos serviços de fisioterapia.

Revista da Rede APS 2019

Método: Estudo quantitativo realizado com 32 pacientes atendidos pelo Publicada em: programa no município de Coromandel-MG, entre janeiro e fevereiro de 2018. A $24 / 07 / 2019$ coleta de dados deu-se por aplicação do questionário sociodemográfico e da identificação da satisfação dos pacientes quanto ao atendimento fisioterapêutico. A análise dos dados foi feita de forma descritiva, com auxílio do software SPSS, versão 18.0.

Resultados: Os entrevistados possuíam média de 72,2 anos de idade, viviam em união estável, com primeiro grau incompleto, renda familiar entre 1-3 salários mínimos advindos da aposentadoria, juntamente com benefícios da previdência social; sem plano de saúde. Uma pequena parte fazia duas ou mais sessões semanais de fisioterapia, sendo a maior demanda neurológica. Os fisioterapeutas realizam um atendimento com gentileza e que respeitam à privacidade durante 0 atendimento, mas que são insuficientes.

Conclusões: Mesmo com os empecilhos encontrados, a satisfação geral relativa à experiência com a fisioterapia foi considerada entre boa a excelente.

DOI:10.14295/aps.v1i2.30

Keren Cristina Rodrigues Soares (Centro

Universitário do Cerrado Patrocínio - UNICERP, Brasil), Juliana Gonçalves Silva de Mattos (Centro Universitário do Cerrado Patrocínio - UNICERP, Brasil);

Correspondência para: Keren Cristina Rodrigues Soares, kerencristina_2011@hot mail.com

Palavras-chave: Satisfação do paciente. Atenção Primária à Saúde. Serviços de Saúde. Fisioterapia. Saúde Pública. 


\section{INTRODUÇÃO}

A criação e a implantação do Sistema Único de Saúde (SUS) em 1988 tornou-se um marco na reorganização dos modelos assistenciais, principalmente com a criação da Estratégia de Saúde da Família (ESF), em 1994, e da Atenção Básica ( $A B$ ), em 2006, ampliando os serviços de saúde a todas as classes sociais (BRASIL, 2006; VIACAVA et al., 2018). A ideia de reorientação do modelo assistencial de saúde baseou-se na criação de equipes multiprofissionais diferentes daquelas formações mínimas (composto por médico, equipe de enfermagem, agentes comunitários de saúde e equipe de saúde bucal), objetivando uma prestação de cuidados diferenciados às populações com características e necessidades específicas e garantindo a integralidade da assistência e reinterando os princípios e diretrizes do SUS (BARBOSA et al., 2010).

O Núcleo de Apoio à Saúde da Família (NASF), criado em 2008, veio para aumentar a abrangência de atendimento e resolubilidade da $A B$ (BRASIL, 2006). Como parte integrante dessa equipe, o fisioterapeuta vem ocupando espaço cada vez maior, tendo sua demanda em crescente expansão, mesmo ainda sendo visto por grande parte da população apenas como reabilitador (FREITAS; BRASIL, 2016).

Os desafios para a inclusão de um profissional pertencente a uma classe relativamente nova (reconhecida no final do século $\mathrm{XIX}$ ) são reais; contudo, a aproximação da teoria e da prática do fisioterapeuta às políticas públicas primárias está acontecendo gradativamente, tornando-se necessário investigar a satisfação dos pacientes com o referido atendimento na atenção primária. Realizar esse estudo faz-se relevante para avaliar se as políticas públicas são realidade em um município de pequeno porte do interior do país, sendo pautadas nas necessidades desse público em consonância com as diretrizes do SUS, em âmbito municipal.

Dessa forma, objetivou-se avaliar a satisfação dos pacientes que são atendidos por fisioterapeutas do
NASF de um município do interior de Minas Gerais (MG).

\section{Materiais e Métodos}

Estudo descritivo, quantitativo, de delineamento transversal, realizado com pacientes cadastrados na Estratégia Saúde da Família (ESF) de Coromandel-MG, que estavam sob atendimento fisioterapêutico, entre janeiro e fevereiro de 2018. Incluíram-se pacientes de ambos os sexos, maiores de 18 anos e que passaram, no mínimo, por três sessões de fisioterapia e excluíram-se pacientes com incapacidades mentais e/ou dificuldades de compreensão e da participação no estudo, assim como aqueles impossibilitados por qualquer prejuízo funcional que não permitiram aos cuidadores/familiares a responderem o questionário.

Foi realizado uma visita domiciliar, uma semana após a derradeira sessão, para a coleta dos dados, explicando-se os objetivos da pesquisa e deixandoos à vontade para participarem ou não, podendo desistir quando lhes for oportuno, sem nenhum prejuízo moral e/ou de atendimento.

Os possíveis riscos aos pacientes foram minimizados por meio da identificação dos mesmos com letras alfabéticas sequenciais, respeitando a resolução 466/2012.

Por fim, foram entrevistados 32 pacientes $(80,0 \%)$, por meio de um questionário sociodemográfico e outro para identificar a satisfação com o atendimento fisioterapêutico recebido adaptado do modelo de Mendonça; Guerra (2007), sendo possível identificar satisfação do paciente com o atendimento fisioterapêutico. $A$ análise foi feita de forma descritiva. A pesquisa foi aprovada por Comitê de Ética sob número 2017/1450 FIS 001.

\section{RESUltados}

Dos participantes identificou-se uma maior média de idade nos homens ( $x=72,8$ anos; $\pm 15,2 ; 46-96$ anos) do que nas mulheres ( $x=71,6$ anos; $\pm 13,3$; 39-93 anos). A maioria viviam em união estável $(56,2 \%)$, com primeiro grau incompleto $(59,4 \%)$ e sem plano de saúde $(87,5 \%)$. A renda familiar 


\section{APS em Revista}

Vol. 1, n. 2, p. 152/161 | Maio/Agosto - 2019

ISSN 2596-3317 - DOI 10.14295/aps.v1i2.30

Soares, K. C. R.; Mattos, J. G. S.

variava de 1 a 3 salários mínimos (100,0\%), advindos da aposentadoria (75,0\% dos homens; $87,5 \%$ das mulheres) e/ou dos benefícios do INSS $(18,7 \%)$.

Quanto à relação paciente-atendimento, observouse uma maior procura por serviços de neurologia e um número reduzido de atendimentos semanais (TAB. 1).

TABELA 1 - Distribuição dos participantes quanto à experiência com a fisioterapia, sobre o reconhecimento do seu diagnóstico e sobre a especialidade que está sendo atendido. Coromandel-MG, 2018.

\begin{tabular}{|c|c|c|c|c|c|c|c|}
\hline \multirow[t]{2}{*}{ Variáveis } & & \multicolumn{2}{|c|}{ Masculino } & \multicolumn{2}{|c|}{ Feminino } & \multicolumn{2}{|c|}{ TOTAL } \\
\hline & & (N) & (\%) & (N) & (\%) & (N) & (\%) \\
\hline Primeira experiência com & Sim & 09 & 56,2 & 08 & 50 & 17 & 53,1 \\
\hline a fisioterapia? & Não & 07 & 43,7 & 08 & 50 & 15 & 46,8 \\
\hline Você sabe qual é o seu & Sim & 15 & 93,7 & 15 & 93,7 & 30 & 93,7 \\
\hline diagnóstico clínico? & Não & 01 & 6,2 & 01 & 6,2 & 02 & 6,2 \\
\hline \multirow{5}{*}{$\begin{array}{l}\text { Especialidade do } \\
\text { tratamento fisioterápico }\end{array}$} & Ortopedia /Traumatologia & 03 & 18,7 & 07 & 43,7 & 10 & 31,2 \\
\hline & Neurologia & 09 & 56,2 & 07 & 43,7 & 16 & 50,0 \\
\hline & Respiratória & 02 & 12,5 & 02 & 12,5 & 04 & 12,5 \\
\hline & Não sabe & 01 & 6,2 & 00 & - & 01 & 3,1 \\
\hline & Outros & 04 & 25,0 & 01 & 6,2 & 05 & 15,6 \\
\hline \multicolumn{8}{|l|}{ Número de sessões por } \\
\hline \multirow[t]{2}{*}{ semana pelo NASF/SUS } & 01 & 13 & 81,2 & 13 & 81,2 & 26 & 81,2 \\
\hline & 02 ou mais & 03 & 18,7 & 03 & 18,7 & 06 & 18,7 \\
\hline \multicolumn{8}{|l|}{ Número de sessões } \\
\hline particulares por semana & 02 ou mais sessões & 02 & 12,5 & 02 & 12,5 & 04 & 12,5 \\
\hline
\end{tabular}

Fonte: Dados da pesquisadora. 2018. 


\section{APS em Revista}

Vol. 1, n. 2, p. 152/161 | Maio/Agosto - 2019

ISSN 2596-3317 - DOI 10.14295/aps.v1i2.30

Soares, K. C. R.; Mattos, J. G. S.

As maiores médias do questionário de qualidade do atendimento foram identificadas nas questões relacionadas à gentileza dos profissionais $(x=4,68)$ e ao respeito à privacidade durante $o$ atendimento $(x=4,62)$. Inferiu-se menores médias à satisfação com a quantidade de atendimentos fisioterapêutico (x=1,90) (QUA. 01).

QUADRO 01 - Distribuição das respostas quanto ao questionário de qualidade do atendimento. Coromandel-MG, 2018.

\begin{tabular}{|c|c|c|c|c|}
\hline & Questões & Respostas & $\mathrm{n}(\%)$ & Média ( \pm ) \\
\hline 1 & $\begin{array}{l}\text { Explicações oferecidas pelo fisioterapeuta com clareza sobre o seu } \\
\text { tratamento no primeiro contato? }\end{array}$ & $\begin{array}{l}\text { Péssimo } \\
\text { Ruim } \\
\text { Bom } \\
\text { Ótimo } \\
\text { Excelente }\end{array}$ & $\begin{array}{l}01 \\
08 \\
13 \\
04 \\
06\end{array}$ & $\begin{array}{c}3,18 \\
( \pm 1,11)\end{array}$ \\
\hline 2 & Segurança transmitida pelo fisioterapeuta durante o tratamento. & $\begin{array}{l}\text { Bom } \\
\text { Ótimo } \\
\text { Excelente }\end{array}$ & $\begin{array}{l}07 \\
05 \\
20\end{array}$ & $\begin{array}{c}4,40 \\
( \pm 0,83)\end{array}$ \\
\hline 3 & Esclarecimento sobre suas dúvidas pelo fisioterapeuta. & $\begin{array}{l}\text { Ruim } \\
\text { Bom } \\
\text { Ótimo } \\
\text { Excelente }\end{array}$ & $\begin{array}{l}01 \\
05 \\
09 \\
17\end{array}$ & $\begin{array}{c}4,31 \\
( \pm 0,85)\end{array}$ \\
\hline 4 & Gentileza do fisioterapeuta. & $\begin{array}{l}\text { Ótimo } \\
\text { Excelente }\end{array}$ & $\begin{array}{l}10 \\
22\end{array}$ & $\begin{array}{c}4,68 \\
( \pm 0,47)\end{array}$ \\
\hline 5 & Privacidade respeitada durante sua sessão de fisioterapia. & $\begin{array}{l}\text { Bom } \\
\text { Ótimo } \\
\text { Excelente }\end{array}$ & $\begin{array}{l}01 \\
10 \\
21\end{array}$ & $\begin{array}{c}4,62 \\
( \pm 0,55)\end{array}$ \\
\hline 6 & Oportunidade dado pelo fisioterapeuta para expressar sua opinião. & $\begin{array}{l}\text { Bom } \\
\text { Ótimo } \\
\text { Excelente }\end{array}$ & $\begin{array}{l}03 \\
10 \\
20\end{array}$ & $\begin{array}{c}4,56 \\
( \pm 0,61)\end{array}$ \\
\hline 7 & Facilidade na marcação das sessões após indicação de fisioterapia? & $\begin{array}{l}\text { Péssimo } \\
\text { Ruim } \\
\text { Bom } \\
\text { Ótimo } \\
\text { Excelente }\end{array}$ & $\begin{array}{l}03 \\
08 \\
08 \\
09 \\
04\end{array}$ & $\begin{array}{c}3,09 \\
( \pm 1,20)\end{array}$ \\
\hline 8 & $\begin{array}{l}\text { Disponibilidade de horários convenientes para realização do seu } \\
\text { tratamento? }\end{array}$ & $\begin{array}{l}\text { Péssimo } \\
\text { Ruim } \\
\text { Bom } \\
\text { Ótimo } \\
\text { Excelente }\end{array}$ & $\begin{array}{l}01 \\
01 \\
11 \\
08 \\
11\end{array}$ & $\begin{array}{c}3,84 \\
( \pm 1,05)\end{array}$ \\
\hline 9 & $\begin{array}{l}\text { A quantidade de vezes que você é atendido pelos serviços de fisioterapia } \\
\text { é satisfatória? }\end{array}$ & $\begin{array}{l}\text { Péssimo } \\
\text { Ruim } \\
\text { Bom } \\
\text { Ótimo } \\
\text { Excelente }\end{array}$ & $\begin{array}{l}14 \\
10 \\
06 \\
01 \\
01\end{array}$ & $\begin{array}{c}1,90 \\
( \pm 1,02)\end{array}$ \\
\hline 10 & Satisfação geral da sua experiência com a fisioterapia. & $\begin{array}{l}\text { Ruim } \\
\text { Bom } \\
\text { Ótimo } \\
\text { Excelente }\end{array}$ & $\begin{array}{l}02 \\
07 \\
12 \\
11\end{array}$ & $\begin{array}{c}4,00 \\
( \pm 0,91)\end{array}$ \\
\hline
\end{tabular}

Fonte: Dados da pesquisadora. 2018. 
A relação paciente-terapeuta foi, em sua maioria, considerada entre boa à excelente. No entanto, algumas questões obtiveram respostas insatisfatórias, como quanto as clareza das explicações referentes ao tratamento no primeiro contato paciente-profissional (péssimo - 3,1\% e ruim - $25,0 \%$ ) e quanto ao esclarecimento das dúvidas pelo profissional (ruim - 3,1\%).

Ao acesso ao atendimento fisioterapêutico pôdese, também, observar uma boa satisfação, mesmo alguns participantes classificando negativamente a facilidade de marcação das sessões após a indicação do tratamento fisioterapêutico (péssimo - 9,4\%; ruim - 25,0\%) e a disponibilidade de horários convenientes para a realização das sessões (péssimo e ruim $-3,1 \%$, respectivamente).

A satisfação geral relativa à experiência com a fisioterapia foi considerada de boa a excelente, mesmo com 6,2\% dos entrevistados tendo-a classificado como ruim. Contudo, a insatisfação foi notória quanto ao número de atendimentos (péssimo - 43,7\%; ruim - 31,2\%).

Avaliou-se o questionário da satisfação do paciente com o atendimento fisioterapêutico, segundo as médias e o desvio padrão de cada domínio. O primeiro domínio relaciona-se à interação entre o paciente-fisioterapeuta (questões 1 a 6 ); 0 segundo domínio está relacionado ao acesso de atendimento fisioterapêutico (questões 7 e 8); o terceiro domínio refere-se à satisfação geral do atendimento (questões 9 e 10).

Apesar do questionário não apresentar um ponto de corte específico para realizar uma classificação da satisfação do atendimento, pode-se inferir uma boa relação entre paciente-profissional ( $X=4,29$; DP- 0,56), com uma boa acessibilidade aos profissionais $(X=3,46 ; \quad D P=0,53)$ e com uma insatisfação ao atendimento $(X=2,95 ; D P=1,48)$.

\section{DisCUSSÃo}

O NASF tem como público de atendimento os mais variados tipos de indivíduos, de diversas faixas etárias e patologias/acometimentos diferentes. A procura pelos serviços de saúde tem aumentado significativamente nos últimos anos, principalmente pelas mulheres que possuem o hábito de se cuidar mais, de forma cultural (SILVA; MENANDRO, 2014). O baixo índice da procura masculina pelos serviços de saúde atribui-se à dificuldade de aderir-se a prática do autocuidado, que segundo eles é associado à fraqueza e a fragilidade, muitas vezes atribuindo ao sexo feminino (VIANA et al., 2014).

No presente estudo os participantes indicaram uma igualdade de gênero na busca pelos serviços, talvez pela amostra ter sido escolhida aleatoriamente ou pela média de idade avançada dos participantes ( $\geq 70$ anos), podendo caracterizar um primeiro motivo-influenciador na avaliação da satisfação do atendimento.

Fisiologicamente, na terceira idade é comum o surgimento de patologias/consequências naturais advindas da maturidade/longevidade, justificando o alto índice de procura pelos serviços de saúde, devido às consequências naturais do envelhecimento (LEVORATO et al., 2014). Assim, pode-se atribuir a satisfação do paciente à expectativa do tratamento, principalmente quando relacionado à duração do tratamento, ao contato físico durante o atendimento e a interação paciente-profissional (SUDA; UEMURA; VELASCO, 2009).

Pesquisar alguns dados sociodemográficos vem auxiliar a análise dos instrumentos utilizados neste estudo, assim como identificar o perfil dos indivíduos que buscam o atendimento público. Também podem auxiliar no planejamento das atividades de saúde realizadas, sendo determinantes na construção de um protocolo de atendimento/tratamento individualizado para cada paciente (FORNASARI; MEDEIROS, 2008).

A maioria da amostra foi composta por participantes de baixo status socioeconômico, com 
renda familiar entre um e três salários mínimos, refletidos no baixo índice de acesso aos serviços particulares, inferindo um segundo motivoinfluenciador na avaliação da satisfação, já que suas expectativas não são grandes por não terem alternativas. $\mathrm{Na}$ sua humildade se sentem beneficiados mesmo que não seja um serviço de boa qualidade (BEATTIE et al., 2002). Esses dados se relacionam aos do estudo de Santos; Bicalho; Silva-Borges (2012) com pacientes atendidos pela clínica-escola de Fisioterapia da Universidade Católica de Brasília. Contudo, identificaram-se também alguns pacientes com nível superior, de ambos os sexos, indicando a participação de todas as classes socioeconômicas enfatizando o princípio de integralidade e equidade do SUS inserido nas políticas do NASF.

A coleta de dados ter sido realizada na residência do próprio paciente, informalmente, por meio de entrevista, pode influenciar alguns participantes que mesmo não estando satisfeitos com alguns quesitos do atendimento se mantiveram passivos quanto às queixas e reclamações, talvez pelo sentimento de dependência de tal atendimento ou medo de perderem o mesmo. Autores afirmam que situações desse modo podem influir diretamente na resposta ao tratamento, deixando os pacientes constrangidos no momento de falar o que realmente sentem, guardando para si sentimentos de vulnerabilidade (GOLDSTEIN; ELLIOTT; GUCCIONE, 2000).

As diretrizes do NASF refletem a atuação do fisioterapeuta na área de promoção, prevenção e reabilitação (MICHELS et al., 2013). Porém, observa-se que grande parte dos casos encaminhados à fisioterapia do NASF são para área de reabilitação onde nem sempre resultam na solução dos problemas, não sendo observado situações de caráter preventivo ou de promoção, por falta de conhecimento e divulgação das demais áreas de atuação (SOUZA et al., 2013).

$\mathrm{Na}$ pesquisa sobre a satisfação do atendimento fisioterapêutico alguns pacientes tiveram contato com a fisioterapia pela primeira vez somente após a doença já instalada. Porém, para parte destes não é o primeiro contato, retratando uma recidiva do tratamento. Se por um lado o atendimento domiciliar permite um conhecimento mais intrínseco do paciente e da família, por outro pode ser um fator prejudicial no atendimento pela falta de recursos e materiais adequados, sendo necessários improvisos.

Dentre os entrevistados um se destacou afirmando que não conhecia seu diagnóstico, indicando falta de esclarecimento e/ou conhecimento, dificultando o resultado do tratamento, pois o mesmo não verá resultados. O Código de Ética da Fisioterapia, artigo 14 , parágrafo $\mathrm{V}$, relata que é dever do fisioterapeuta informar ao paciente ou responsável sobre o diagnóstico, os objetivos do tratamento e demais procedimentos a serem executados (COFFITO, 2013).

Com relação à prevalência das patologias encontradas, identificou-se maior demanda em ortopedia/traumatologia e em neurologia. As mulheres tendem desenvolver mais doenças musculoesqueléticas devido às funções domésticas e profissionais que exercem (SANTOS et al., 2017), assim como os homens tendem a desenvolver mais doenças neurológicas, como o Acidente Vascular Encefálico (AVE) (ARAUJO et al., 2017), corroborando com achados de outros estudos (MATIAS; ALENCAR; SILVA, 2011; SANTOS; BICALHO; SILVA-BORGES, 2012).

Em relação ao número de atendimento fisioterapêutico semanal, mais de $80,0 \%$ dos pacientes têm atendimento apenas uma vez por semana, o que dificulta a evolução do tratamento, levando muitos à desistência, onerando o SUS e enfatizando ainda mais o caos instalado no sistema de saúde brasileiro (ACIOLE; OLIVEIRA, 2017).

No referido município do estudo, atualmente há duas fisioterapeutas que realizam os atendimentos pelo NASF, em domicílio, com uma lista de espera considerável, o que pode justificar o número de atendimentos semanais, e um alto índice de insatisfação quanto ao número de atendimentos $(X=1,9)$. Mesmo assim, no questionário foi considerado que as profissionais são gentis, respeitam a individualidade de cada paciente ao atendimento, apresentando uma boa satisfação 
com o atendimento em relação à interação paciente-profissional, com regularidade ao acesso e moderada satisfação com o atendimento.

O estudo ampliou a visão de uma sociedade preocupada em reabilitar e não prevenir, reforçando a importância da conscientização da população quanto à prevenção. A relação pacienteterapeuta foi considerada o ponto forte do estudo e um fator importante para que o NASF seja bem sucedido no município, já que a parte humanitária destaca-se para uma boa qualidade no atendimento, principalmente na faixa etária em que a amostra do estudo se encontra.

Devido o NASF ainda estar em processo de construção, foram encontrados algumas fragilidades como a falta de conhecimento das áreas de atuação da fisioterapia, a falta de estruturas e materiais para o trabalho, poucos profissionais capacitados para trabalhar na $A B$, se equiparando à realidade nacional e contribuindo para o baixo nível da qualidade nos serviços prestados e, consequentemente, na baixa satisfação dos pacientes (SOUZA; CALVO, 2018).

Também foram encontradas poucas referências sobre o assunto. Os principais estudos foram realizados no início da criação do NASF, impossibilitando assim a comparação dos resultados, por exemplo, após 10 anos de atuação. Assim, a necessidade de mais pesquisas para auxiliar na compreensão das políticas públicas e na estruturação das equipes, além de identificar se os ideais do programa são colaborativos para o sistema de saúde ou não, fazem-se pertinentes.

O baixo número da amostra não compromete 0 estudo e é justificável pela metodologia utilizada (de delineamento transversal), onde todos os pacientes atendidos no recorte do tempo foram convidados a participar da pesquisa. O número de atendimentos fisioterapêuticos no município é influenciado pelo baixo contingente de fisioterapeutas no NASF $(n=2)$ e pela escassez de equipamentos e materiais.

\section{CONCLUSÃO}

O objetivo do estudo foi alcançado com sucesso pois foi possível evidenciar uma satisfação geral relativa à experiência com a fisioterapia entre boa a excelente. As divulgações das ações do NASF devem ser ampliadas para que suas propostas se tornem conhecidas, principalmente em relação à atuação da fisioterapia nas áreas primárias, quebrando o estigma de reabilitador.

O acesso da população aos serviços fisioterapêuticos foram, de certa forma, facilitados, mesmo com todos os empecilhos encontrados.

Estudos que buscam identificar a satisfação dos pacientes atendidos fazem-se relevante, pois pode ser considerados indicadores da assistência fornecida, fundamental para a avaliação do programa e para a qualidade do atendimento.

\section{REFERÊNCIAS BIBLIOGRÁFICAS}

(1) BRASIL. Ministério da Saúde. A construção do SUS: histórias da Reforma Sanitária e do Processo Participativo. Brasília: MS, 2006.

(2). VIACAVA, F.; OLIVEIRA, R. A. D.; CARVALHO, C. C.; LAGUARDIA, J.; BELLIDO, J. C. SUS: oferta, acesso e utilização de serviços de saúde nos últimos 30 anos. Revista Ciência e Saúde Coletiva, v. 23, n. 6, p. 1751-1762, 2018 Disponível em: http://www.scielo.br/pdf/csc/v23n6/1413-8123csc-23-06-1751.pdf. Acesos em: 20 novembro 2018.

(3). BARBOSA, E. G.; FERREIRA, E. G.; FERREIRA, D. L. S.; FURBINO, S. A. R. Experiência da Fisioterapia no Núcleo de Apoio à Saúde da Família em Governador Valadares, MG. Revista Fisioterapia em Movimento, v. 23, n. 2, p. 323 - 330, 2010. Disponível em: http://www.scielo.br/scielo.php?pid=S010351502010000200015\&script=sci_abstract\&tlng=pt Acesso em: 13 junho 2018.

(4). FREITAS, M. J.; BRASIL, A. M. R. Potencialidades e desafios da fisioterapia no contexto da Atenção Primária à Saúde: análise documental. Revista Saúde em Redes, v. 2, n. 3, p. 262-272, 2016. 


\section{APS em Revista}

Vol. 1, n. 2, p. 152/161 | Maio/Agosto - 2019

ISSN 2596-3317 - DOI 10.14295/aps.v1i2.30

Soares, K. C. R.; Mattos, J. G. S.

Disponível

em: http://revista.redeunida.org.br/ojs/index.php/redeunida/article/view/730 Acesso em: 25 maio 2018.

(5). MENDONÇA, K. M. P. P.; GUERRA, R. O. Desenvolvimento e validação de um instrumento de medida da satisfação do paciente com a fisioterapia. Revista Brasileira de Fisioterapia, v. 11, n. 5, p. 369-376, 2007. Disponível em: http://www.scielo.br/pdf/rbfis/v11n5/a07v11n5.p df Acesso em: 20 maio de 2018.

(6). SILVA, S. P. C.; MENANDRO, M. C. S. As representações sociais da saúde e de seus cuidados para homens e mulheres idosos. Revista Saúde e Sociedade, v. 23, n. 2, p. 626-640, 2014. Disponível em: http://www.scielo.br/pdf/sausoc/v23n2/01041290-sausoc-23-2-0626.pdf Acesso em: 10 junho 2018.

(7). VIANA, J, R, S.; RAIOCOVITCH, T.; GOLIAS, A. R.; PETERNELLA, F. M. N.; MENDES, F. C.; GRABOWSKI, J. Avaliação da satisfação com a fisioterapia de pacientes atendidos em uma clínica escola de Maringá - PR. Revista UNINGÁ, v. 17, n. 2, p. 16, $2014 . \quad$ Disponível em: http://revista.uninga.br/index.php/uningareviews/ article/view/1493 Acesso em: 11 julho 2018.

(8). LEVORATO, C. D.; MELLO, L. M.; SILVA, A. S.; NUNES, A. A. Fatores associados à procura por serviços de saúde numa perspectiva relacional de gênero. Revista Ciência e Saúde Coletiva, v. 19, n. 4, p. 1263-1274, 2014. Disponível em: http://www.scielo.br/scielo.php?pid=S1413$81232014000401263 \&$ script=sci_abstract\&tlng=pt Acesso em: 11 julho 2018.

(9). SUDA, E. Y.; UEMURA, M. D.; VELASCO, E. Avaliação da satisfação dos pacientes atendidos em uma clínica-escola de Fisioterapia de Santo André, SP. Revista Fisioterapia e Pesquisa, v. 16, n. 2, p. 126-131, 2009. Disponível em: http://www.scielo.br/pdf/fp/v16n2/06.pdf. Acesso em: 15 junho 2018.

(10). FORNASARI, C.; MEDEIROS, F. D. Perfil dos pacientes pediátricos atendidos na clínica escola de fisioterapia (CEF). 2008. Monografia Universidade do Sul de Santa Catarina, Tubarão -
SC. Disponível em: http://fisiotb.unisul.br/Tccs/08b/caiano/ARTIGO.pdf. Acesso em: 20 maio de 2018.

(11). BEATTIE, P. F.; PINTO, M. B.; NELSON, M. K.; NELSON, R. Patient satisfaction with outpatient physical therapy: instrument validation. Phys Ther, v. 82, n. 6, p. 557-565, 2002. Disponível em: https://academic.oup.com/ptj/article/82/6/557/28 36972 Acesso em: 17 setembro 2018.

(12). SANTOS, J. B.; BICALHO, K. A.; SILVA-BORGES, M. B. Características e nível de satisfação dos pacientes atendidos na clínica-escola de fisioterapia da Universidade Católica de Brasília. Revista EFDeportes.com, v. 17, p. 171, 2012. Disponível em: https://www.efdeportes.com/efd171/satisfacaodos-pacientes-na-clinica-de-fisioterapia.htm Acesso em: 15 junho 2018.

(13). GOLDSTEIN, M. S.; ELLIOTT, S. D.; GUCCIONE, A. A. The development of an instrument to measure satisfaction with physical therapy. Phys Ther, v. 80, n. 9, p. 858-863, 2000. Disponível em: https://www.ncbi.nlm.nih.gov/pubmed/10960933. Acesso em: 10 maio 2018.

(14). MICHELS, R. S.; STINIESKI, M. S.; BRAGA, M. V.; BRAGA, M. S.; BOLZAN, L. M.; GALVÃO, A. A. Y.; BELLINI, M. I. B. Normativas e produção do conhecimento sobre o NASF: elementos para reflexão. 2013. Disponível em: http://ebooks.pucrs.br/edipucrs/anais/sipinf/edico es/l/5.pdf Acesso em: 5 agosto 2018.

(15). SOUZA, M. C.; BOMFIM, A. C.; SOUZA, J. N.; FRANCO, T. B. Fisioterapia e Núcleo de Apoio à Saúde da Família: conhecimento, ferramentas e desafios. O Mundo da Saúde, v. 37, n. 2, p. 176184, 2013. Disponível em: http://bvsms.saude.gov.br/bvs/artigos/mundo_sa ude/fisioterapia_nucleo_apoio_saude_familia.pdf Acesso em: 03 junho 2018.

(16). COFFITO. Conselho Federal de Fisioterapia e Terapia Ocupacional. Resolução no424: estabelece o Código de Ética e Deontologia da Fisioterapia. Brasília: 2013. Disponível em: https://www.coffito.gov.br/nsite/?p=3187 Acesso em: 05 maio 2018. 


\section{APS em Revista}

Vol. 1, n. 2, p. 152/161 | Maio/Agosto - 2019

ISSN 2596-3317 - DOI 10.14295/aps.v1i2.30

Soares, K. C. R.; Mattos, J. G. S.

(17). SANTOS, E. C.; ANDRADE, R. D.; LOPES, S. R. G.; VALGAS, C. Prevalence of musculoskeletal pain in nursing professionals working in orthopedic setting. Rev Dor, v. 18, n. 4, p. 298-306, 2017. Disponível em: http://www.scielo.br/scielo.php?script=sci_arttext \&pid=S1806-00132017000400298.

http://www.scielo.br/scielo.php?script=sci_arttext \&pid=S1806-00132017000400298 Acesso em: 12 junho 2018.

(18). ARAUJO, L. P. G.; SOUZA, G. S.; DIAS, P. L. R.; NEPOMUCENO, R. M.; COLA, C. S. D. Principais fatores de risco para acidente vascular encefálico e suas consequências: uma revisão de literatura. REINPEC, v. 3, n. 1, p. 283-296, 2017. Disponível em:

http://reinpec.srvroot.com:8686/reinpec/index.ph $\mathrm{p} / \mathrm{reinpec} /$ article/view/269 Acesso em: 16 outubro 2018.

(19). MATIAS, K. F. S.; DI ALENCAR, T. A. M.; SILVA, T. C. D. Análise da satisfação do paciente com a fisioterapia na clínica escola de fisioterapia da UEG - ESEFFEGO. Revista Movimenta, v. 4, n. 1, p.. 1932, 2011. Disponível em: https://www.revista.ueg.br/index.php/movimenta /article/view/7084 Acesso em: 10 abril 2018.

(20). ACIOLE, G. G.; OLIVEIRA, D. K. S. Percepções de usuários e profissionais da saúde da família sobre o Núcleo de Apoio à Saúde da Família. Saúde debate, v. 41, n. 115, p. 1090-1101, 2017. Disponível em: http://www.scielo.br/scielo.php?pid=S0103$11042017000401090 \&$ script=sci_abstract \&tIng=pt Acesso em: 12 abril 2018.

(21). SOUZA, T. T.; CALVO, M. C M. Avaliabilidade dos núcleos de apoio à saúde da família com foco na integração às equipes apoiadas. Revista de Saúde Pública, v. 52, n. 41, p. 01-11, 2018. Disponível em: http://www.scielo.br/pdf/rsp/v52/pt_0034-8910rsp-S1518-87872018052000122.pdf Acesso em: 22 janeiro 2019. 


\section{ABSTRACT}

Objective: To evaluate patients' satisfaction with the physiotherapy services.

Methods: A quantitative study was performed with 32 patients attended by the program in the city of Coromandel-MG, between January and February, 2018. Data collection was done by applying the sociodemographic questionnaire and identifying patients' satisfaction regarding physiotherapeutic care. The analysis of the data was done in a descriptive way, using SPSS software, version 18.0 .

Results: The interviewees had a mean of $\mathbf{7 2 . 2}$ years of age, lived in a stable union, with incomplete first degree, family income between 1-3 minimum wages from retirement, together with social security benefits; without health insurance. A small part did two or more weekly sessions of physiotherapy, with the greatest neurological demand. Physiotherapists perform care with kindness and respect privacy during care, but are insufficient.

Conclusions: Despite the obstacles encountered, overall satisfaction with physical therapy experience was considered good to excellent.

Keywords: Patients Satisfaction. Primary Health Care. Health Services. Physical Therapy Specialty. Public Health. 